\title{
FABRICATION OF SUPERMEMBRANES AND OTHER STRUCTURES USING ONE-STEP POROUS POLYSILICON FILMS
}

\author{
George M. Dougherty, Albert P. Pisano ${ }^{1}$ and Timothy Sands \\ Department of Materials Science and Engineering and ${ }^{1}$ Department of Mechanical Engineering \\ Berkeley Sensor and Actuator Center, 497 Cory Hall, University of California - Berkeley 94720
}

\begin{abstract}
We have recently developed a simple and controlled process by which fluid-permeable porous polycrystalline silicon (polysilicon) films can be created in a single ten-minute LPCVD deposition, using only standard equipment, with no other processing steps required. The remarkable combination of qualities exhibited by these films, coupled with their great ease of use, allows the easy microfabrication of structures that were never before feasible.
\end{abstract}

\section{INTRODUCTION}

The fabrication of large freestanding membranes, sealed cavities, and similar structures has long been a difficult task for MEMS designers and process developers. Backside wafer through-etching with anisotropic wet etchants, and similar techniques, have generally been required, often with the addition of wafer-to-wafer bonding and other involved processes.

Recently, as a result of extensive materials science investigations [1], we have discovered a process by which polycrystalline silicon (polysilicon) films can be deposited in a porous condition, such that they are permeable to liquid etchant solutions. The phenomenon of "permeable polysilicon" was first observed several years ago [2], but the origin of the permeability and the means of process control were unknown. The recent investigations led to the discovery of a growth regime for polysilicon whereby standard low-pressure chemical vapor deposition (LPCVD) methods result in a polysilicon film that is porous in the as-deposited state, requiring no additional process steps. The process is very simple, requiring only a single tenminute deposition step under the correct process conditions.

The new One-Step Porous Polysilicon process offers a powerful new tool for microfabrication. Open etch holes, or backside access, are not required in order to release a porous polysilicon film because etch solution can pass through the film itself. As a result, a whole range of microfabricated structures that would have been difficult or impossible to make using traditional micromachining techniques can now be made quickly and easily.

\section{SUPERMEMBRANES}

A dramatic example of the capability offered by the new process is the fabrication of unbroken, free-standing membranes of remarkable size ("supermembranes"), only $100 \mathrm{~nm}$ thick, aligned over their entire area only 1 micron above the underlying substrate. Such membranes have been produced which are larger than a square centimeter in size. The fabrication requires only the deposition of a porous film over a sacrificial oxide, which is subsequently released though the porous film using HF, a release process which takes less than a minute.

The porous polysilicon process yields films that are inherently almost free of internal residual stress, as described below, and are generally used in a slightly (less than $50 \mathrm{MPa}$ ) tensile condition for membrane applications. Perhaps even more important for applications is the fact that they are naturally aligned with high precision over the substrate, separated only by the thickness of the removed sacrificial layer. These membranes immediately suggest new possibilities for acoustic microphones, high-sensitivity pressure sensors, and other MEMS devices. A photo of part of a supermembrane is shown in figure 1 .

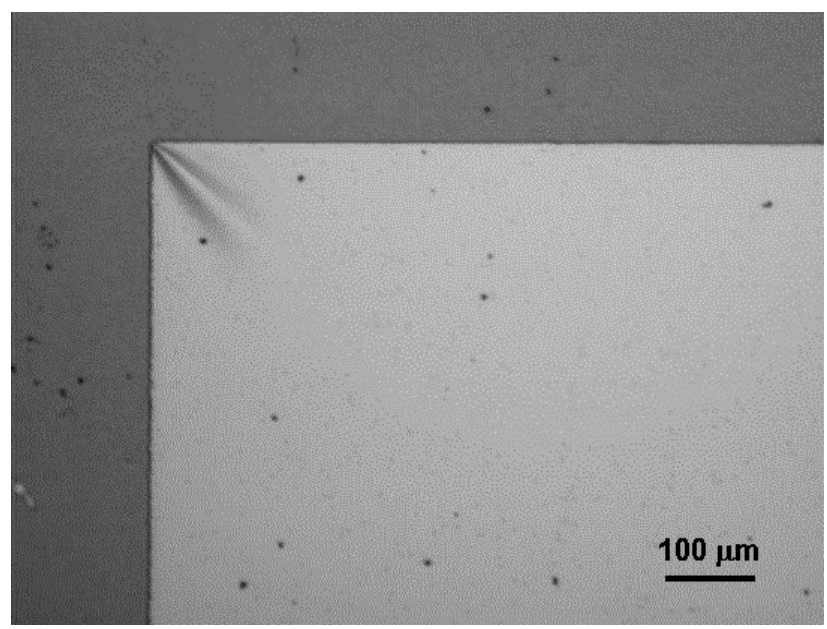

Figure 1. One corner of a very large $(0.9 \mathrm{~cm} \times 1.2 \mathrm{~cm})$ porous polysilicon supermembrane. The pinching or "bedsheeting" visible in the sharp corner is an optical effect seen in released tensile porous polysilicon membranes.

\section{OTHER EXAMPLE STRUCTURES}

The porous polysilicon films are highly conformal, have very low residual stress, and are composed entirely of a standard CMOS material (silicon). They are resistant to stiction, such that many structures with dimensions less than $100 \mu \mathrm{m}$ can be released without the use of critical point drying or similar techniques. In addition, the pores can be easily sealed with a subsequent layer of silicon nitride, polysilicon, or other material, to produce a hermetically sealed membrane. This process has been used in our laboratory to fabricate micromachined ultrasonic immersion transducers. A wide range of other structures has been fabricated using the films, a selection of which is shown here.

Because the porous films are conformal, almost any sacrificial structure can be coated with a porous layer and then released to yield a hollow structure with walls composed of porous polysilicon. An example of this is shown in figure 2, a broken cross-section of a porous polysilicon cantilever beam. Other examples of new structures that are possible are the dual-lumen microchannel structure fabricated on a silicon dioxide substrate, 
shown in figure 3 , and the enclosed serpentine flow channel made entirely of porous polysilicon, shown in figure 4. One-Step Porous Polysilicon offers a convenient way of making porous fluidic structures for separation, filtration, and other purposes.

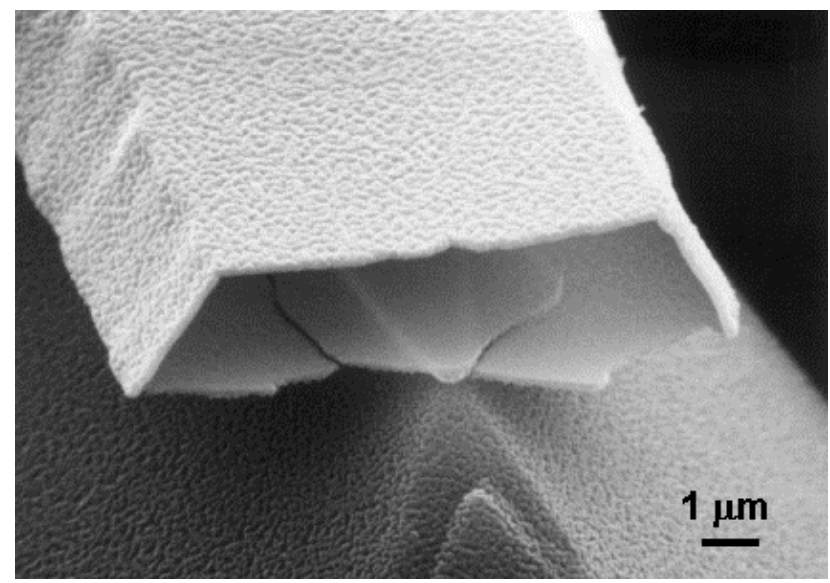

Figure 2. A porous polysilicon cantilever beam, broken open to show the hollow interior.

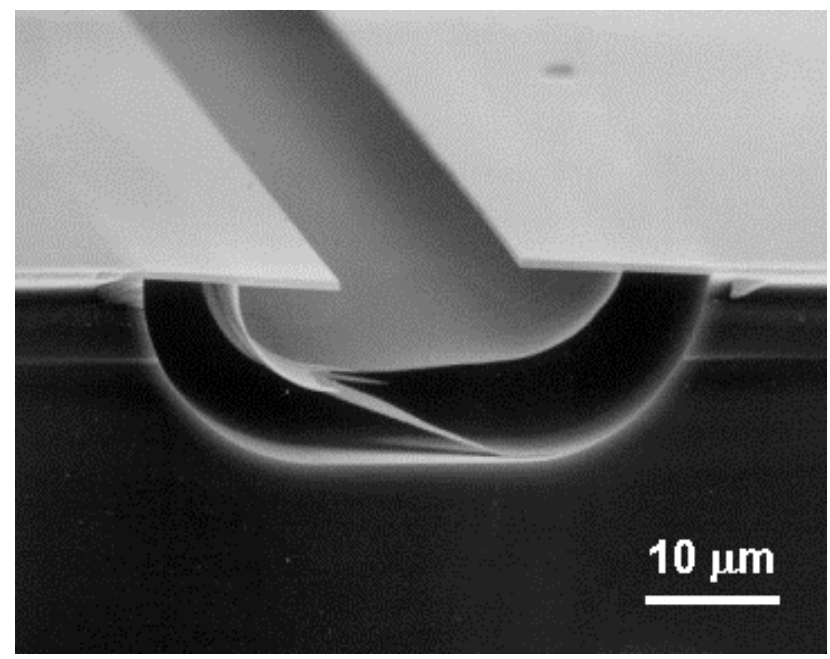

Figure 3. A microchannel in a silicon dioxide substrate, featuring two interior fluid passages separated by a curved layer of porous polysilicon.

\section{ONE-STEP POROUS POLYSILICON}

The pores in the polysilicon material occur spontaneously during deposition within a narrow process window. The distinctive cobblestone morphology characteristic of the porous films occurs at the transition between a lower temperature deposition regime that yields tensile films, and a higher temperature regime that yields compressive films. By measuring film residual stress using standard wafer-curvature methods, the process is easily tuned and controlled to produce porous films with high yield. Figure 5 is a high magnification SEM photo showing the pores between polysilicon grains, typically ranging from 10 to
$50 \mathrm{~nm}$ in size. More information on the process is available in reference [1].

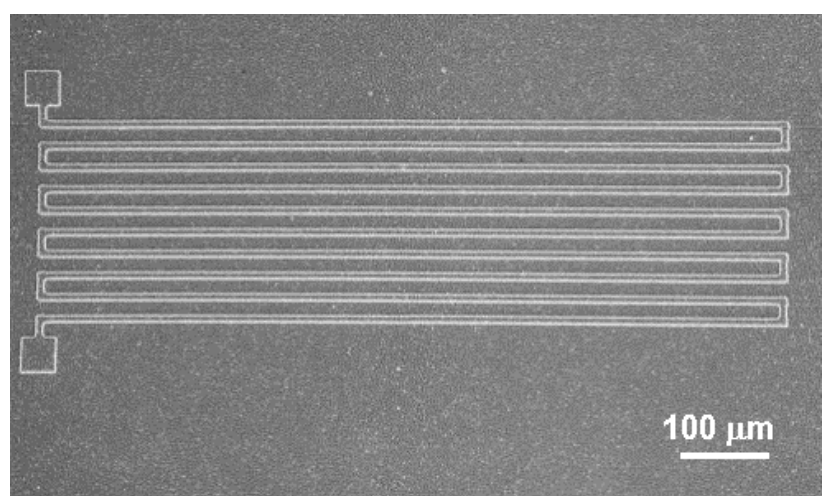

Figure 4. A serpentine hollow structure with walls made of porous polysilicon. Porous structures of this size can be released without the use of critical point drying or similar special techniques.

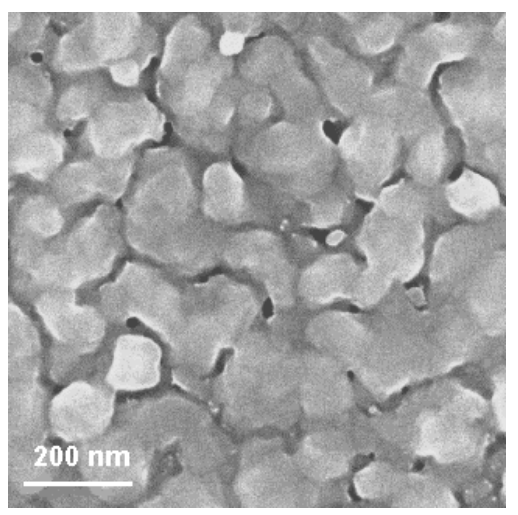

Figure 5. SEM photo of the structure of a One-Step Porous Polysilicon film, showing the pores between grains.

\section{CONCLUSION}

The One-Step Porous Polysilicon material offers attractive new opportunities for MEMS designers and fabrication process developers. Its combination of properties and its great convenience and ease of use allow the simple and elegant fabrication of a wide range of structures, a few simple examples of which have been presented here.

\section{REFERENCES}

1. G.M. Dougherty, T. Sands, and A. Pisano, "The Materials Science of 'Permeable Polysilicon' Thin Films," in Materials Science of Microelectromechanical Systems (MEMS) Devices IV, Materials Research Society vol. 687, Boston, MA, November 2001.

2. L. Lin, K. M. McNair, R.T. Howe, and A.P. Pisano, "Vacuumencapsulated Lateral Microresonators," Proc. 7th International Conf. On Solid-State Sensors and Actuators (Transducers 93), Yokohama, Japan, June 7-10 1993, pp. 270-3. 